\title{
Tako-Tsubo-Kardiomyopathie durch Marihuana?
}

Bei der als Tako-Tsubo benannten Stress-Kardiomyopathie handelt es sich um ein erst vor etwa 20 Jahren beschriebene akute schwere Funktionsstörung des Herzmuskels, deren Symptomatik einem akuten Koronarsyndrom ähnelt. Auslöser sind häufig emotionale Belastungen, betroffen sind vorwiegend ältere Frauen.

Das Krankheitsbild scheint aber auch bei jüngeren Marihuana-Konsumenten vorzukommen. Diese sollten wissen, dass sie schnell zum Arzt gehen sollten, wenn sie plötzlich Brustschmerzen oder Atemnot empfinden, berichteten Autoren vom St. Luke's University Health Network in Bethlehem, Pennsylvania.

Sie hatten Daten von 33.343 Patienten untersucht, die in USA-weit mit der Diagnose Stress-Kardiomyopathie hospitalisiert worden waren. 210 hatten angegeben oder wurden anhand von Urinproben identifiziert, dass sie Marihuana konsumiert hatten. Die Patienten waren vergleichsweise jung und wiesen wenige kardiovaskuläre Risikofaktoren auf. Dennoch waren ihre Risiken höher, einen Herzstillstand $(2,4$ vs. $0,8 \%)$ oder eine gravierende Rhythmusstörung ( 2,4 vs. $0,6 \%)$ zu erleiden, welche die Implantation eines Defibrillators erforderlich machte.

Marihuana-Konsumenten hatten deutlich häufiger Depressionen, Psychosen und Angststörungen durchgemacht. Sie konsumierten auch mehr Alkohol, Tabak und andere Drogen als Stressmyopathie-Patienten ohne Marihuana-Konsum. Allerdings hatten die Autoren in ihrer retrospektiven Analyse diese Faktoren berücksichtigt und darauf adjustiert. $\quad D E$
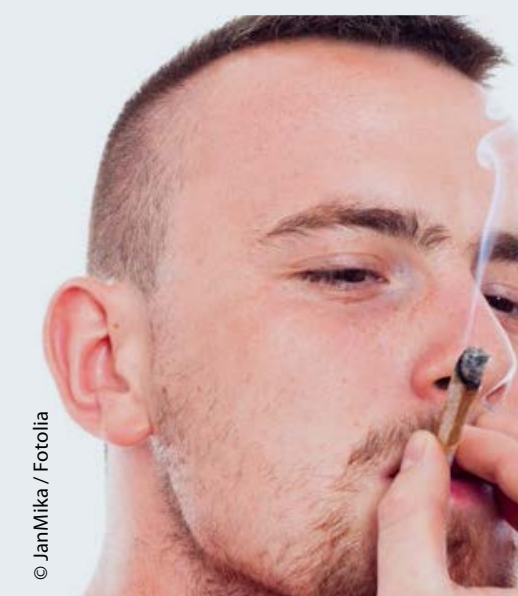

,

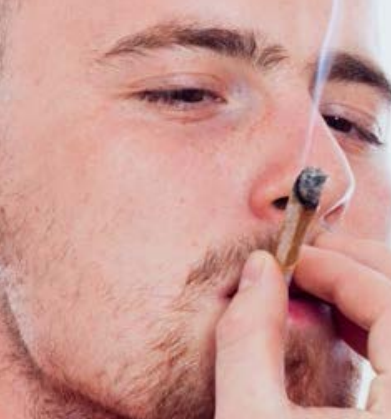

ließ kein gutes Haar an Nissens randomisierter PRECISION-Studie und verwies auf zahlreiche Schwächen.

\section{Celecoxib, Ibuprofen und Naproxen im Vergleich}

An der PRECISION-Studie hatten 24.081 Patienten mit Osteoarthrose (90\%) oder rheumatoider Arthritis (10\%) teilgenommen, die eine analgetische Dauertherapie benötigten. Laut Einschlusskriterien sollten sie ein erhöhtes kardiovaskuläres Risiko aufweisen. Tatsächlich hatten drei Viertel entsprechende Risikofaktoren, aber nur ein Viertel war kardiovaskulär erkrankt.

Die Patienten wurden entweder mit Celecoxib $(2 \times 100 \mathrm{mg} / \mathrm{d})$, Ibuprofen $(3 \times$ $600 \mathrm{mg} / \mathrm{d})$ oder Naproxen $(2 \times 375 \mathrm{mg} / \mathrm{d})$ behandelt. Bei nicht ausreichender Wirksamkeit konnte die Dosis von Ibuprofen und Naproxen erhöht werden, für Celecoxib hingegen ging das in der Regel nicht, weil in vielen Ländern $200 \mathrm{mg}$ die Tageshöchstdosis darstellt. Letztlich betrugen die mittleren Tagesdosen für Celecoxib $209 \mathrm{mg}$, für Ibuprofen $2.045 \mathrm{mg}$ und für Naproxen $852 \mathrm{mg}$. Alle Patienten nahmen zusätzlich mit Esomeprazol ein Magenschutzpräparat ein; $45 \%$ standen zu Beginn unter niedrigdosierter ASS-Medikation.

Die Patienten wurden im Schnitt 34 Monate nachverfolgt. Primäres Ziel der von Pfizer finanzierten Studie war es, die Nichtunterlegenheit von Celecoxib gegenüber den Vergleichssubstanzen bezüglich der kardiovaskulären Sicherheit darzustellen. Dies gelang.

\section{Nicht mehr Komplikationen}

Nissen stellte die Ergebnisse sowohl nach dem Intention-to-Treat-Prinzip als auch nach einer On-Treatment-Analyse dar. Letztere sei zur Erfassung von tatsächlichen Nebenwirkungen sinnvoll.

In beiden Analysen wiesen Patienten unter Ibuprofen numerisch die meisten kardiovaskulären Komplikationen auf und Patienten unter Celecoxib die wenigsten. Statistisch war Celecoxib eindeutig nicht unterlegen $(\mathrm{p}<0,001)$.

Bei den laut Nissen „hypothesengenerierenden" sekundären Endpunkten schnitt Celecoxib gut ab. In der OnTreatment-Analyse verzögerte es die Zeit bis zu schweren kardiovaskulären

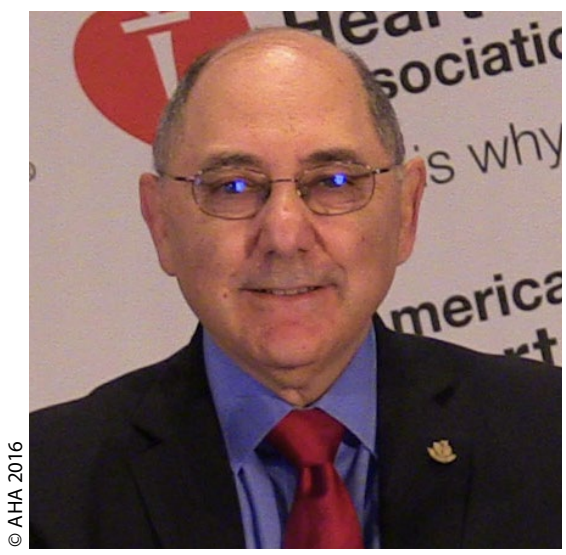

AHA-Past-Präsident Elliott Antman ging mit der PRECISION-Studie hart ins Gericht.

Ereignissen (HR: 0,82), die Zeit bis zum Herzkreislauftod (HR: 0,64) und die Zeit bis zum Tod jeder Ursache (HR: $0,68)$ signifikant gegenüber Ibuprofen. In der ITT-Analyse zeigte Naproxen gegenüber Celecoxib ein Signal zu erhöhter Mortalität $(\mathrm{p}=0,052)$.

\section{Weniger gastrointestinale und renale Nebenwirkungen}

Schwere gastrointestinale Nebenwirkungen traten unter beiden Vergleichsmedikamenten trotz Protonenpumpen-Hemmer-Prophylaxe mit erheblich höherer Frequenz auf. Ähnliches galt für schwere renale Nebenwirkungen: Hier war Celecoxib Ibuprofen in beiden Analysen und Naproxen in der On-Treatment-Analyse überlegen. Die Ergebnisse seien unabhängig davon, ob der Patient ASS einnahm, so Nissen.

\section{Zahlreiche Schwächen}

Die Studie habe aber Schwächen, räumte Nissen ein: $69 \%$ der Patienten stoppten ihre analgetische Medikation oder setzten diese um. $27 \%$ konnten nicht bis Studienende nachverfolgt werden. Dies sei üblich bei Schmerzpatienten.

Darüber hinaus handle es sich nicht wirklich um ein kardiovaskuläres Risikokollektiv. Die Studie lasse keine Rückschlüsse zu, wie bei Herzpatienten zu verfahren sei. Schließlich sei Celecoxib mit im Schnitt $209 \mathrm{mg} / \mathrm{d}$ moderat dosiert worden. Die Studie zeige die relative Sicherheit der drei untersuchten Medikamente, aber nicht die von 20 weiteren in der Apotheke erhältlichen NSAR. 